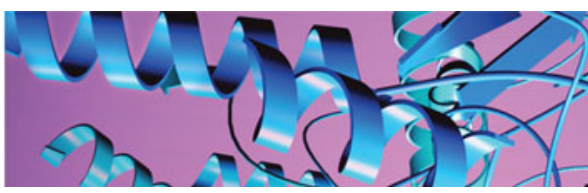

\title{
Quantum entanglement: facts and fiction - how wrong was Einstein after all?
}

\author{
Bengt Nordén*
}

Chair Professor of Physical Chemistry, Chalmers University of Technology, SE-41296 Gothenburg, Sweden

Quarterly Reviews of Biophysics (2016), 49, e17, page 1 of 13 doi:10.1017/S0033583516000111

Dedicated in memory of Ahmed H. Zewail

Abstract. Einstein was wrong with his 1927 Solvay Conference claim that quantum mechanics is incomplete and incapable of describing diffraction of single particles. However, the Einstein-Podolsky-Rosen paradox of entangled pairs of particles remains lurking with its 'spooky action at a distance'. In molecules quantum entanglement can be viewed as basis of both chemical bonding and excitonic states. The latter are important in many biophysical contexts and involve coupling between subsystems in which virtual excitations lead to eigenstates of the total Hamiltonian, but not for the separate subsystems. The author questions whether atomic or photonic systems may be probed to prove that particles or photons may stay entangled over large distances and display the immediate communication with each other that so concerned Einstein. A dissociating hydrogen molecule is taken as a model of a zero-spin entangled system whose angular momenta are in principle possible to probe for this purpose. In practice, however, spins randomize as a result of interactions with surrounding fields and matter. Similarly, no experiment seems yet to provide unambiguous evidence of remaining entanglement between single photons at large separations in absence of mutual interaction, or about immediate (superluminal) communication. This forces us to reflect again on what Einstein really had in mind with the paradox, viz. a probabilistic interpretation of a wave function for an ensemble of identically prepared states, rather than as a statement about single particles. Such a prepared state of many particles would lack properties of quantum entanglement that make it so special, including the uncertainty upon which safe quantum communication is assumed to rest. An example is Zewail's experiment showing visible resonance in the dissociation of a coherently vibrating ensemble of NaI molecules apparently violating the uncertainty principle. Einstein was wrong about diffracting single photons where space-like anti-bunching observations have proven recently their non-local character and how observation in one point can remotely affect the outcome in other points. By contrast, long range photon entanglement with immediate, superluminal response is still an elusive, possibly partly misunderstood issue. The author proposes that photons may entangle over large distances only if some interaction exists via fields that cannot propagate faster than the speed of light. An experiment to settle this ' $i n$ teraction hypothesis' is suggested.

In 1935, Einstein, Podolsky and Rosen (EPR) presented a paradox, which seemed to imply a fundamental discrepancy between quantum and classical mechanics and which they meant proves that the former is not a 'complete theory' (Einstein et al. 1935). Einstein (1936) elaborated on the theme with a more nuanced description later, in what sense he considers quantum mechanics (QM) incomplete. His concern that it is the way QM is applied to singular

* Author for Correspondence: B. Nordén, Chair Professor of Physical Chemistry, Chalmers University of Technology, SE-41296 Gothenburg, Sweden. E-mail: norden@chalmers.se

Editor: A. Warshel particulate systems rather than whether it is a correct theory, was my inspiration for revisiting these grounds and for trying to assess whether there is yet any concrete experimental support for the hypothesis that quantum entanglement could provide a means by which long-range communication between particles or photons of single entangled pairs can occur. While Einstein appears incorrect in claiming that QM is an underdetermined theory, he has clearly made several valid points about its applicability to real systems.

Einstein (1936) says 'Die Schrödinger-Gleichung bestimmt die zeitlichen Änderungen, welche die System-Gesamtheit erfärhrt, sei es ohne, sei es mit äusseren Einwirkungen auf 
das Einzel-System.' He was concerned by the simplification of using one wave function to describe a single-particle system and, furthermore, that it may be prone to artifacts, due to weaker, disregarded but still potentially significant, perturbing interactions with the surroundings, if the predicted expectation is not adequately integrated with respect to all parameters involved. This has often been taken to be equivalent to requiring 'hidden parameters', but an alternative interpretation would be to say that the Hamiltonian must be complete with respect to all relevant particles and interactions involved (including interactions with the measuring apparatus), and that the wave function refers to a large number of identically prepared experiments.

The discussion led to a philosophically important debate between Einstein and Bohr (Bohr, 1935a, b) in which Einstein's standpoint was seen as fundamentally deterministic. This is not quite true as his probabilistic approach could allow for considerable freedom. His main objection was against the concept of 'entanglement' (Verschränkung), suggested by Schrödinger (1935), and to which the EPR paradox paper was a retort: Einstein could not accept the idea that two distant, non-interacting systems, jointly described by one wave function, could be correlated in such a way that a change in the state of one results in an immediate response of the other, by (as he puts it) some 'spooky action at a distance'.

'Immediate' specifically means a violation of the principle that the speed of light sets the upper limit for how fast the separated particles may communicate with each other. Phrased in other words, by 'locality' Einstein meant that, for two remotely separated non-interacting systems, a complete description of physical reality implies that any action performed on one system must not affect the description of the other, or put bluntly: entanglement cannot work over large distances! Much of the EPR paper is logical and reasonable. For example, a two-particle system is assumed to be characterized by a difference of $x$ coordinates, $x_{1}-x_{2}$, and the sum of the $\mathrm{x}$-components of their momenta, $p_{1 x}+$ $p_{2 x}$, which is fully consistent with the QM formalism as follows from the commutativity between $x_{1}-x_{2}$ and $p_{1 x}+p_{2 x}$. The first experimental demonstration of the physics of EPR was made by Kimble's group ( $\mathrm{Ou}$ et al. 1992a, b), which later led to theoretical criteria for unambiguous verification of true entanglement (Duan et al. 2000; Simon, 2000).

An immediate critique of the EPR paper by Kemble (1935) identifies 'physical reality' as the key issue. He suggests the statistical ensemble interpretation of Slater as a way to resolve the EPR paradox, but this seems to have overlooked the fact that the expansion of the wave function $\Psi\left(x_{1}, x_{2}\right)$ of the combined system is not unique (see below). Kemble later, after receiving a copy of an unpublished manuscript by Podolsky (Jammer, 1974) retracted his accusation that the EPR paper contained logical errors. Bohr (1935b) argued, elegantly using a Gedankenexperiment with a double- slit diaphragm, that freedom of choice, to measure either $p_{1}$ or $x_{1}$, involves a discrimination between different and mutually exclusive experiments, in conflict with EPR. He stated that the result of a quantum measurement, because of the unlimited divisibility of events, reflects on the wholeness of the system rather than on the state of the isolated objects.

The most commonly cited case of entanglement is that of Bohm \& Aharonov (1957), complemented by a paper by Bell (1966): a pair of spin one-half particles are formed somehow in the singlet state and move in opposite directions. We imagine a zero total spin molecule consisting of two atom fragments, each with spin one-half, i.e. denoting spin angular momentum components $S_{z}=+$ or $-(1 / 2)(h / 2 \pi)$. Consider the simplest of all such molecules, viz. a hydrogen molecule, $\mathrm{H}_{2}$, which we let homolytically split into two hydrogen atom radicals: $\mathrm{H}_{2}->2 \mathrm{H}$. The wave function describing the whole system, the original molecule as well as the separated atoms A and $\mathrm{B}$, is then:

$\Psi=2^{-1 / 2}\left[\Psi_{+}(1) \Psi_{-}(2)-\Psi_{-}(1) \Psi_{+}(2)\right]$

where $\Psi_{+}(1)$ refers to the wave function of the atomic state in which one electron (1) has electron spin $+1 / 2$, etc. After splitting of the molecule, in an adiabatic way that does not affect the spin, the two free hydrogen radicals $\mathrm{H}$. are free to diffuse away from each other. If one could measure the spin of one of the atoms, one should immediately be able to conclude that the spin of the other atom is opposite to that of the first. The problem is the lack of robustness of the wave function to describe a single entangled pair of particles. Once one of the particles is touched, e.g. by the magnetic field of a Stern-Gerlach detector, the whole wave function will collapse, into $\Psi_{-}(1) \Psi_{+}(2)$ or $\Psi_{+}(1) \Psi_{-}(2)$, virtually independent of how far the particles have travelled away from each other.

In this context, Bell's theorem is relevant (Bell, 1966). It sets out to discriminate between the quantum mechanical (entanglement) and classical (local realism) models. By assuming a fixed hidden-parameter framework in which he calculates expectation values for the spin components of a pair of spin-half particles, Bell shows that regardless of the choices that are made for certain unspecified functions, the expectation values obey a certain inequality. We shall return to this inequality in the case of 'entangled' photons and indicate some inconsistency, possibly related to Bell's assumption about what is 'classical logic'.

The general question we may ask is whether any perturbation of the spin of atom A can somehow instantaneously alter the spin of atom B, irrespective of an arbitrary (large) distance separation? (In support of the conclusion we shall reach, no direct experiment has so far unambiguously shown that it can, in case A and B are not interacting any longer with each other). The question is crucial in several contexts. If the answer is ' $n o$ ', this may have profound consequences for many proposed applications that are based on the 
expectation that long-range entanglement is a real and useful phenomenon. Applications include quantum communication (Gisin \& Thew, 2007), quantum cryptography (Ekert, 1991) and quantum 'teleportation' (Bennett et al. 1993).

We consider below both the case of the split hydrogen molecule, where we specifically address the distance and time dependence of a possible quantum entanglement, and the analogous case of entangled photons. ${ }^{1}$

Bohm \& Aharonov (1957) suggested that the EPR paradox could be experimentally tested using photons by creating a state describing two photons given off simultaneously, with opposite angular momenta (circular polarizations) - and in Eq. (1) having plus instead of minus sign (because photons are bosons). Later treatments have sometimes taken the photon pair to have either identical parallel or orthogonal linear polarizations. The most notable experiments have been carried out by Freedman, Clauser, Gisin, Kimble, Horne, Mandel, Zeilinger, Aspect and Hansen (Aspect et al. 1982a, b; Clauser et al. 1969; Clauser \& Horne, 1974; Clauser \& Shimony, 1978; Freedman \& Clauser, 1972; Giustina et al. 2015; Guerreiro et al. 2012; Hensen et al. 2015; Horne \& Zeilinger, 1985; Kimble et al. 1977; Weihs et al. 1998; Zbinden et al. 2001).

For the hydrogen radical pair system there does indeed exist a distance and time dependence (in agreement with Einstein's intuition). Although this does not decouple the wave function, it will lead to the latter's attenuation in the limit of infinite separation and infinite time in such a way that it will not, for practical purposes, exhibit any remaining correlation except possibly for what we may call a 'memory' effect - when appropriate statistical averages have been taken over time and number of observations. Such a memory effect trivially reflects the fact that $\mathrm{A}$ and $\mathrm{B}$ at the point of dissociation have a complementary bias. That is, if atom A may be shown somehow to have spin $+1 / 2$ then we know that atom $B$, when sent off into space, had spin $-1 / 2$, and vice versa. If $\mathrm{QM}$ works still at a distance (i.e. if imperfections such as due to depolarization noise in fiber etc. can be excluded) a measurement-limited catch will still remain, so we cannot decide which case we have without collapsing the wave function.

That the spin of a single confined electron may lose memory of direction, in typically $10^{-6} \mathrm{~s}$, has been detected in quantum dots (Johnson et al. 2005). With a statistical interpretation, the memory concept may involve hidden parameters and thus only first after an appropriate statistical average is taken, can a metric correlation between spins be read

1 'Photon' was coined by chemist Gilbert N. Lewis and physicist Frithiof Wolfers in 1926 to denote a quantum particle of light. Lewis' theory of shared pair of electrons making up a covalent bond is an anticipation of the later quantum mechanical Valence Bond theory of Heitler and London that we apply here for $\mathrm{H}_{2}$. out. For a swarm of initially even incoherent photons, field interactions may create self-coherence at higher intensities. An example of single-spin coherence are the oscillations observed in fluorescence of a molecule after exposure to an radio frequency field pulse that mixes the spin sublevels and starts an evolution making the spins and emitted photons coherent (Wratchtrup et al. 1993). All the same, as we shall conclude below, the effect of distance and time in practical contexts is to gradually blur entanglement.

\section{Distance and time dependence of an atomic entangled state}

We may write a (spatial) electron wave function of a hydrogen-like molecule as (Griffiths, 2008):

$\Psi=2^{-1 / 2}\left[\phi_{\mathrm{A}}\left(\mathbf{r}_{1}\right) \phi_{\mathrm{B}}\left(\mathbf{r}_{2}\right) \pm \phi_{\mathrm{B}}\left(\mathbf{r}_{1}\right) \phi_{\mathrm{A}}\left(\mathbf{r}_{2}\right)\right.$

where $\phi_{\mathrm{A}}\left(\mathbf{r}_{1}\right)$ is the spatial wave function for the first electron (position vector $\mathbf{r}_{1}$ ) and $\phi_{\mathrm{B}}\left(\mathbf{r}_{2}\right)$ for the second electron etc. The symmetric (plus sign) form of Eq. (2a) typically represents the lower energy, bound state of an electron, enhancing the electron density in the middle between the two atoms A and $\mathrm{B}$. To include spin we must enforce the requirement that the overall product wave function for space and spin is antisymmetric under particle exchange. This means that Eq. (2a) must be multiplied by the antisymmetric (minus) or symmetric (plus) combination of electron spin wave functions:

$2^{-1 / 2}[\alpha(1) \beta(2) \pm \alpha(2) \beta(1)]$

where $\alpha$ means spin $+1 / 2$ and $\beta$ means spin $-1 / 2$. Using perturbation theory, the exchange interaction ('exchange' meaning interchange of spatial or spin coordinates of identical particles) in the hydrogen molecule is found to have two possible energies (van Vleck 1932):

$E_{ \pm}=E_{0}+\left(C \pm J_{e x}\right) /\left(1 \pm B^{2}\right)$

where $E_{+}$is the spatially symmetric case and $E_{-}$the spatially antisymmetric case. $B$ is the overlap integral $\left\langle\phi_{\mathrm{A}}\left(r_{1}\right) \phi_{\mathrm{B}}\left(r_{1}\right)\right\rangle$, $C=\left\langle\phi_{\mathrm{A}}\left(r_{1}\right)^{2} H^{e x} \phi_{\mathrm{B}}\left(r_{2}\right)^{2}\right\rangle$ is the Coulomb integral and $J_{e x}=$ $\left\langle\phi_{\mathrm{A}}\left(r_{1}\right) \phi_{\mathrm{B}}\left(r_{2}\right) H^{e x} \phi_{\mathrm{A}}\left(r_{1}\right) \phi_{\mathrm{B}}\left(r_{2}\right)\right\rangle$ the exchange integral, where the latter two depend on the inverse distance between the two nuclei $\left(R_{\mathrm{AB}}^{-1}\right)$.

When spin is included, the total wave function is either the $(+)(-)$ or the $(-)(+)$ combinations of wave functions $(2 \mathrm{a})$ and $(2 b)$, corresponding to the spin singlet and triplet states of the hydrogen molecule, respectively. The triplet state is triply degenerate in the absence of external fields, with the three spin wave functions being $\alpha(1) \alpha(2), \beta(1) \beta(2)$, and $2^{-1 / 2}[\alpha(1) \beta(2)+\alpha(2) \beta(1)]$. The energy separation (Dirac, 1926; White, 2007)

$E_{+}-E_{-}=2\left(J_{\text {ex }}-C B^{2}\right) /\left(1-B^{4}\right)$

falls off linearly with $J_{e x}$ and thus approximately as $R_{\mathrm{AB}}^{-1}$ with the proton-proton separation. $J_{e x}$ in fact decreases even 
more rapidly as the centers of $\phi_{\mathrm{A}}$ and $\phi_{\mathrm{B}}$ move apart and experience less interactive overlap with each other $-C$ and $B$ then also vanish. Note that while this description, which is the (covalent) Valence Bond model, is only approximate at short $R_{\mathrm{AB}}$, it becomes a more exact description at large inter-nuclear separations, which is the case we are interested in.

When the energy difference between the singlet and the triplet states of the two separate hydrogen radicals goes to zero, any perturbation due to the surrounding universe could lead to a complete contamination of states, and annihilation of any entanglement.

In the complete absence of any other matter or external fields the zero angular momentum of the singlet state $2^{-1 / 2}[\alpha(1) \beta(2)-\alpha(2) \beta(1)]$ will be conserved so that each hydrogen radical will keep its respective spin angular momentum, whether $\alpha$ or $\beta$, for ever. However, with a QM interpretation, i.e. preserved full wave function, it is first at measurement (collapse of wave function) it is decided which particle will have $\alpha$ and which $\beta$. In all practical experiments there will be some surrounding matter (gas, liquid or solid state) that could cause admixture of singlet and triplet states: any external perturbation may thus give rise to a spin change, for example, via some spin-lattice kind of relaxation mechanism (Atkins et al. 1973; Ardavan et al. 2007). Here temperature, reflecting somehow the system's degree of excitation, and the fact that there is generally a radiation field present associated with a certain temperature, can be an important parameter (Ninham \& Daicic, 1998; Wennerstrom et al. 1999) - higher temperature observed to shorten the relaxation time. Typically in a molecular magnet system an electronic $T_{1}$ relaxation time of $10^{6} \mathrm{~ns}$ at $2 \mathrm{~K}$ may drop to $10^{2} \mathrm{~ns}$ at $10 \mathrm{~K}$ and so on, relaxation time empirically varying as $T^{-1}$ (Bogani \& Wernsdorfer, 2008). In the solid state, the characteristic time for establishing thermal equilibrium between the lattice and the spin of ferromagnetic gadolinium was estimated to $0 \cdot 1 \mathrm{~ns}$ at $45 \mathrm{~K}$ (Vaterlaus et al. 1991). Experiments with nitrogen-vacancy (N-V) centers in diamond indicate that $\mathrm{N}-\mathrm{V}$ center ensembles may have spincoherence times as long as $50 \mu$ s at room temperature (Epstein et al. 2005), but generally in liquids relaxation varies as $T^{-1}$, typically from $0 \cdot 1 \mathrm{~ns}$ at $-40^{\circ} \mathrm{C}$ to $0.01 \mathrm{~ns}$ at $50^{\circ} \mathrm{C}$ (Zager \& Freed, 1982).

Another problem is that it is difficult to imagine how the dissociation energy, hugely exceeding the spin flip energy, may be delivered gently without affecting the spin. A vanishing entanglement may not exclude the possibility of some residual 'magnetic memory' of the original spin remaining, for example, if implanted into some solid state material, and potentially possible to pick up by sensitive instrumentation. It would, in principle, be anti-correlated, between the two hydrogen fragments for each split event. However, only in a passive, exclusive way, will there be any residual memory and, furthermore, spin-ups and spin-downs will statistically be at random for an ensemble of split 'unsorted' hydrogen atoms. The ensemble picture contrasts the putative dynamic correlation of an entangled pair of spin particles, which was the origin of Einstein's concern. Entanglement with $30 \mathrm{~s}$ lifetime of trapped ions has been demonstrated by Blatt \& Wineland (2008).

Wennerström and Westlund have considered the problem of randomizing spin during passage of a Stern-Gerlach magnet (Wennerström \& Westlund, 2013). They draw attention to an issue with Bell's theorem, the derivation of which was based on the assumption of only two possible spin values after exiting the analyzer. Their arguments for a wide spin distribution are supported by considering the $T_{2}$ spin relaxations due to spin angular momentum transfer between particles and magnet, results that are found to be in agreement with the blurred pattern in original Stern-Gerlach data.

Some ground breaking discoveries on atoms and ions should be noted. Wineland and his group (Rowe et al. 2001; Turchette et al. 1998) demonstrated violation of Bell's inequality with material particles, using ${ }^{9} \mathrm{Be}^{+}$ions prepared in Bell-like singlet and triplet states, driven by stimulated Raman processes from pairs of laser beams in one of two geometries, an important parallel to the photon experiments that we shall discuss later. In a topical review Leggett (2002) discusses limits of QM and specifically over what length scales QM has been tested - he points into some directions where QM might potentially break down, one being very small length scales (very high energies) and another, which could be also chemically and biophysically interesting, being short times and space-like separation in the sense of special relativity.

\section{How Einstein (possibly) was thinking}

The problem of entanglement is closely connected with the experiment. The entangled state has no meaning until somebody tries to probe it - then it is like opening a box to find out what spin one of the hydrogen radicals has, and then, puff?, the wave function collapses into one of the eigenstates. With this view, it is the act of making a measurement that forces the system to react irrespective of how far away the two parts are from each other. This is what Einstein saw as a spooky action at a distance! So where is the problem?

Already during the 5th Solvay Conference in 1927, Einstein expresses a view that clearly lies behind the 1935 EPR paper (Jammer, 1974). He considers two viewpoints of behavior for a particle passing a slit, and then hitting a screen:

Viewpoint 1: 'the de Broglie-Schrödinger waves do not represent one individual particle but rather an ensemble of particles distributed in space'... 'Thus $|\Psi(r)|^{2}$ expresses the probability density that there exists at $r$ some particle of the ensemble.' 
Viewpoint 2 ' $\mathrm{QM}$ is considered as a complete theory: each particle moving towards the screen is described as a wave packet which, after diffraction, arrives at a certain point on the screen where $|\Psi(r)|^{2}$ expresses the probability that one and the same particle shows its presence at $r$. As long as no localization has been effected, the particle must be considered as potentially present over the whole area of the screen; however, as soon as it is localized, a peculiar action-at-a-distance must be assumed to take place, which prevents the continuously distributed wave in space from producing an effect at two places on the screen.' He also said: 'the interpretation 2 of $|\Psi(r)|^{2}$, I think, contradicts the postulate of relativity.'

If we apply Einstein's Viewpoint 1 to the dissociating hydrogen molecules, the wave function does not refer to any specific pair of hydrogen atoms but to an ensemble of identically prepared pairs. For instance, if a beam of hydrogen atoms is passed through a Stern-Gerlach apparatus, one of the two emerging beams will be deflected up and contain only atoms with $S_{z}=(+1 / 2) h / 2 \pi$ while the other beam will only contain atoms with $S_{z}=(-1 / 2) h / 2 \pi$. This experiment, which may better be called 'state preparation', as suggested by Bransden \& Joachain (2000), can be immediately repeated by letting, e.g. the $+1 / 2$ beam pass a second Stern-Gerlach magnet oriented in the same way as the first. Then all $+1 / 2$ particles will pass, but none of those corresponding to $-1 / 2$. What is essential to note here, and which applies to how the Bell inequality is interpreted (see below), is that in our experiment we have prepared the beams of atoms in a reproducible way, and the measurement can be repeated again and again. However, by way of contrast, if we were to perform a true measurement on an unprepared object, to assess its energy, position, momentum etc., one has a different situation: about the former (prepared) system we may say something about its future, while for the latter (unknown) system we might only tell something about its immediate past.

Einstein's probabilistic view of reality will require more parameters to be considered in a statistical ensemble system (such as, e.g. temperature). 'Ensemble' is here considered a series of repeated preparations of close to identical individual quantum systems measured sequentially one by one. Assume, for example, that we consider Eq. (1) as a linear superposition of the two wave functions $\Psi_{+}(1) \Psi_{-}(2)$ and $\Psi_{-}(1) \Psi_{+}(2)$. We may continue by including additional pairs of (non-interacting) particles and in this way get some wave function for the whole system

$\Psi($ total $)=N^{-1 / 2} \Sigma\left[\Psi_{+}(1) \Psi_{-}(2)-\Psi_{-}(1) \Psi_{+}(2)\right]$

whose statistical meaning is represented by the square of its modulus:

$$
\begin{aligned}
\mid\left.\Psi(\text { total })\right|^{2}= & (1 / N)\left\{\Sigma\left|\Psi_{+}(1) \Psi_{-}(2)\right|^{2}+\Sigma\left|\Psi_{-}(1) \Psi_{+}(2)\right|^{2}\right. \\
& \left.-2 \Sigma\left|\Psi_{+}(1) \Psi_{-}(2)\right|\left|\Psi_{-}(1) \Psi_{+}(2)\right| \cos \delta\right\}
\end{aligned}
$$

with $\delta$ representing some phase shift reflecting e.g. how the different entanglements were created, their coherence etc. The interference term can obviously have very drastic effects on the total and may represent the statistical distribution of further (hidden?) parameters of the experiment.

Therefore, let us elaborate on the experiment a little further along the lines of Bransden \& Joachain (2000). Let us say we want to measure the value of an observable A of our system. The result of a single measurement must thus be one of the eigenvalues $a_{n}$ of A corresponding to some eigenvector $\left|\phi_{n}\right\rangle$. However, the system cannot be viewed in isolation. The analyzer (Stern-Gerlach apparatus or light polarizer, see below) should be combined with the system we wish to analyze:

$\{$ system + analyzer $\}$

Suppose the initial wave function for the ensemble of states of the two-particle system is $\sum b_{n} \mid \phi_{n}>$ with the expansion running over all pure states of a complete set of states, $\left|\phi_{n}\right\rangle$. The state function for the combined, system plus analyzer, may then be written as the direct product:

$\mid \Psi($ total,$t)\rangle=\Sigma_{n} b_{n}\left|\phi_{n}>\otimes\right| \Phi_{0}>$

with $\left|\Phi_{0}\right\rangle$ being the initial eigenvector of the analyzer. The total state function, we presume, will satisfy some version of the time-dependent Schrödinger equation and evolve so that, after the measurement at time $t^{\prime}$, it will become $\mid \Psi$ (total, $\left.t^{\prime}\right)>$ :

$\mid \Psi\left(\right.$ total, $\left.t^{\prime}\right)>=\Sigma_{m} b_{m}\left|\phi_{m}>\otimes\right| \Phi_{m}>$.

Now Einstein's (putative) interpretation of $\mid \Psi\left(\right.$ total, $\left.\left.t^{\prime}\right)\right\rangle$ could be that within a large ensemble of identically prepared states, one should look for the fraction in which the value $a_{n}$ is found, which will be given by $\left|b_{m}\right|^{2}$, all state vectors assumed normalized to unity. This view is self-consistent and any attempt to interpret Eq. (5b) in terms of a singleparticle system would seem inconsistent and unphysical. The consistency arises from taking the ensemble view. A statistical interpretation of QM by Ballentine (1970), using a minimum of assumptions, arrives at similar recommendations. Below we shall use a function, Malus' law, to introduce a probabilistic view of a many-particle ensemble to entangled photons.

An example of the dissipative evolution of a prepared set of states is the study of femtosecond dynamics of dissociation of sodium-iodide molecules by Zewail and coworkers (Engel et al. 1988; Rose et al. 1988; Zewail, 1988, 1990): during the cycle of a vibration of the molecule after being excited to its first excited state by a femtosecond pulse, the passage of the Landau-Zener crossing point (at inter-nuclear separation $6.93 \AA$ ) could occur either when the covalent quantum wave packet is on its way out (then leading to dissociation into $\mathrm{Na}$ and I atoms) or the ionic wave packet on its way 
in (leading to trapping of $\mathrm{NaI}$ in the bound ground state). Whereas, a single-particle experiment would encounter a problem to resolve the wave packet motion because of the uncertainty principle, the quantum statistics of the large number of molecules coherently excited by a femtosecond pulse, to vibrate all of them in phase, will over-rule the uncertainty and provide macroscopic features to the oscillations, possible to experimentally follow as either activated complex or free atoms, appearing as ringing (resonance) particle emission intensities with coinciding temporal peak positions. An analogous classical case is the ringing that DNA molecules display during migration through an electrophoresis gel, if 'excited' in phase by the sudden application of an electric field (Nordén et al. 1991). In both cases the ringing is sharp in the beginning but eventually blurred out as different molecules (NaI and DNA, respectively) encounter various perturbations and get out of phase.

\section{Bell's theorem and photon entanglement}

The EPR paradox has been elaborated on in Gedankenexperiments by Bohm (1952) and Bell (1966) to show that no hidden-variable theory may reproduce all predictions from QM. Note again, that Einstein believed the predictions of QM to be correct, but only as consequences of appropriate statistical averaging over distributions of (possibly hidden) further variables.

Bell's argument proceeds as follows. In the EPR-Bohm Gedankenexperiment a source emits pairs of spin $1 / 2$ particles from singlet state systems. Alternatively, pairs of photons are produced in an analogous, non-factorizing state. After the particles have separated, one performs correlated measurements of their spins (or polarizations for photons). The detection devices - hereafter polarizers - can have arbitrary settings $\mathbf{a}$ and $\mathbf{b}$, being vectors in the planes, on the respective sides of the source, perpendicular to the photon propagation direction, with any direction in the plane allowed. Let us denote the observed results as ' + ' if the polarization is found parallel with a, and '- ' if the polarization is found perpendicular, etc. Contributing to the total probability $P_{ \pm \pm}(\mathbf{a}, \mathbf{b})$, we have the following four possibilities:

$P_{++}(\mathbf{a}, \mathbf{b}), P_{+-}(\mathbf{a}, \mathbf{b}), P_{-+}(\mathbf{a}, \mathbf{b}), P_{--}(\mathbf{a}, \mathbf{b})$

The function

$$
\begin{aligned}
E(\mathbf{a}, \mathbf{b})= & P_{++}(\mathbf{a}, \mathbf{b})+P_{--}(\mathbf{a}, \mathbf{b})-P_{+-}(\mathbf{a}, \mathbf{b}) \\
& -P_{-+}(\mathbf{a}, \mathbf{b})
\end{aligned}
$$

representing the correlation of the particles' polarizations, was studied by Bell. Considering the particles in each pair and adding locality assumptions he arrived at certain inequalities that could not always be fulfilled by the predictions of QM.
Defining the correlation function

$S=\left|E(\mathbf{a}, \mathbf{b})-E\left(\mathbf{a}, \mathbf{b}^{\prime}\right)\right|+\left|E\left(\mathbf{a}^{\prime}, \mathbf{b}\right)+E\left(\mathbf{a}^{\prime}, \mathbf{b}^{\prime}\right)\right|$

his inequality could be written (Carg \& Mermin, 1987; Clauser et al. 1969; Larsson, 1998):

$S \leqslant(4 / \eta)-2$

with $\eta$ the detection efficiency and where $S$ thus involves four series of repeated measurements involving the polarizers in four different combinations of orientations: (a, b), (a, $\left.\mathbf{b}^{\prime}\right),\left(\mathbf{a}^{\prime}, \mathbf{b}\right)$ and $\left(\mathbf{a}^{\prime}, \mathbf{b}^{\prime}\right)$ (Clauser et al. 1969).

Two critical questions emerge from this analysis:

(1) whether true correlation may be experimentally achieved between the two emitted photons (i.e. no time lag between their emissions), and

(2) whether their polarizations may be considered known as a result of the design of the source.

In the experiments by Freedman \& Clauser (1972) and later Aspect et al. (1982a, b), a Ca ${ }^{40}$ cascade is exploited to generate pairs of photons of different wavelength, assumed to be entangled. This was an EPR type experiment first made by Kocher \& Commins (1967), which unfortunately only set the polarizers at $0^{\circ}$ and $90^{\circ}$ relative to each other - a new experiment and inequality was proposed by Clauser et al. in 1969. A problem, however, was the finite lifetime of the intermediate ${ }^{1} \mathrm{P}_{1}$ state (approximately $5 \mathrm{~ns}$ ) making the second photon lag behind the first one, possibly also depolarized and not entangled. Optical Parametric Amplifiers (OPA) are used today to generate coherent states of photons as developed by Mandel and followers (Friberg et al. 1985; Hong et al. 1987). The temporal and spatial resolution may still be a problem if the two photons are not created at the same time and spot in the crystal. Potentially the high photon flux, required to get high electric fields in the non-linear crystal, could present another problem: which photon forms an entangled pair with which? Two entangled photons should be typically created at times differing less than, say, $10^{-16} \mathrm{~s}$, corresponding to a flight time of $10 \%$ the 'size' of a $300 \mathrm{~nm}$ photon (my tentative suggestion). This aspect has as far as I know not been addressed, but it is possible that the small probability (say, 1 in roughly $10^{12}$ pump photons) for this to occur may be compensated for by the extreme intensities of signal and idler photons in the diffraction-limited volume of the crystal. An attractive aspect of the parametric down conversion experiment is that it emits trains of highly correlated photons in one process - photons that may be measured later far apart.

As for polarization conditions these may vary, but generally the photons created in the non-linear crystal by highintensity electric laser fields will have a well-defined polarization. We will in our own Gedankenexperiment below assume that the two photons have the same linear 
polarization parallel with some laboratory reference axis of the photon-generating crystal. If we were to challenge Bell's theorem it is enough to find a set of (possible) photon parameters that will violate the statement that 'classical' (see Section 7) and quantum results are different at certain angles of polarizer settings.

\section{Classical versus QM correlations}

Following the analysis of Aspect et al. (1982a, b), who essentially repeated the experiments and analysis by Freeman \& Clauser, we may now calculate the expected classical correlation function assuming that all photons, whether quantum entangled or not, all have initially parallel (say vertical) polarization. If we are going to question the validity of the Bell inequality, we can disregard the issue of whether we measure on correlated photons. First let us consider the probabilities $P_{i j}(\mathbf{a}, \mathbf{b})$ for the case of the outcoming photon being vertically polarized using classical optical theory.

According to Malus' empirical, but also theoretically justified law of polarization (discovered by Étienne-Louis Malus in 1808) the intensity of a beam of linearly polarized light is attenuated by an inserted polarizer by the factor $\cos ^{2} \phi$ so that the light intensity before $\left(S_{0}\right)$ and after $(S)$ the polarizer are related as:

$S / S_{0}=\cos ^{2} \phi$

with $\phi$ the angle between the plane of linear polarization and the polarizer axis.

Malus' law immediately tells us the (classical) probability for transmission of a photon through a polarizer. At $0^{\circ}$ and $180^{\circ}$ there is maximum probability (one) for transmission, whereas at $90^{\circ}$ and $270^{\circ}$ the probability that a photon should come through is identically zero. At all angles the probability is given by Eq. (10).

If the polarizer settings (vectors $\mathbf{a}$ and $\mathbf{b}$ ) are set at angles $\alpha_{a}$ and $\alpha_{b}$, respectively, we can now calculate the classical correlation function:

$$
\begin{aligned}
E(\mathbf{a}, \mathbf{b})= & P_{++}(\mathbf{a}, \mathbf{b})+P_{--}(\mathbf{a}, \mathbf{b})-P_{+-}(\mathbf{a}, \mathbf{b}) \\
& -P_{-+}(\mathbf{a}, \mathbf{b}) \\
E(\mathbf{a}, \mathbf{b})= & {\left[\left\langle\cos ^{2} \alpha_{a} \cos ^{2} \alpha_{b}\right\rangle+\left\langle\sin ^{2} \alpha_{a} \sin ^{2} \alpha_{b}\right\rangle\right.} \\
& \left.-\left\langle\cos ^{2} \alpha_{a} \sin ^{2} \alpha_{b}\right\rangle-\left\langle\sin ^{2} \alpha_{a} \cos ^{2} \alpha_{b}\right\rangle\right] \\
& /\left[\left\langle\cos ^{2} \alpha_{a} \cos ^{2} \alpha_{b}\right\rangle+\left\langle\sin ^{2} \alpha_{a} \sin ^{2} \alpha_{b}\right\rangle+\left\langle\cos ^{2} \alpha_{a} \sin ^{2} \alpha_{b}\right\rangle\right. \\
& \left.+\left\langle\sin ^{2} \alpha_{a} \cos ^{2} \alpha_{b}\right\rangle\right]
\end{aligned}
$$

If we assume that each and every count is averaged for each $E(\mathbf{a}, \mathbf{b})$ at a time, we have (the normalizing denominator obviously becomes equal to unity):

$$
\begin{aligned}
\langle E(\mathbf{a}, \mathbf{b})\rangle= & \left\langle\cos ^{2} \alpha_{a}\left(\cos ^{2} \alpha_{b}-\sin ^{2} \alpha_{b}\right)-\sin ^{2} \alpha_{a}\left(\cos ^{2} \alpha_{b}\right.\right. \\
& \left.\left.-\sin ^{2} \alpha_{b}\right)\right\rangle
\end{aligned}
$$
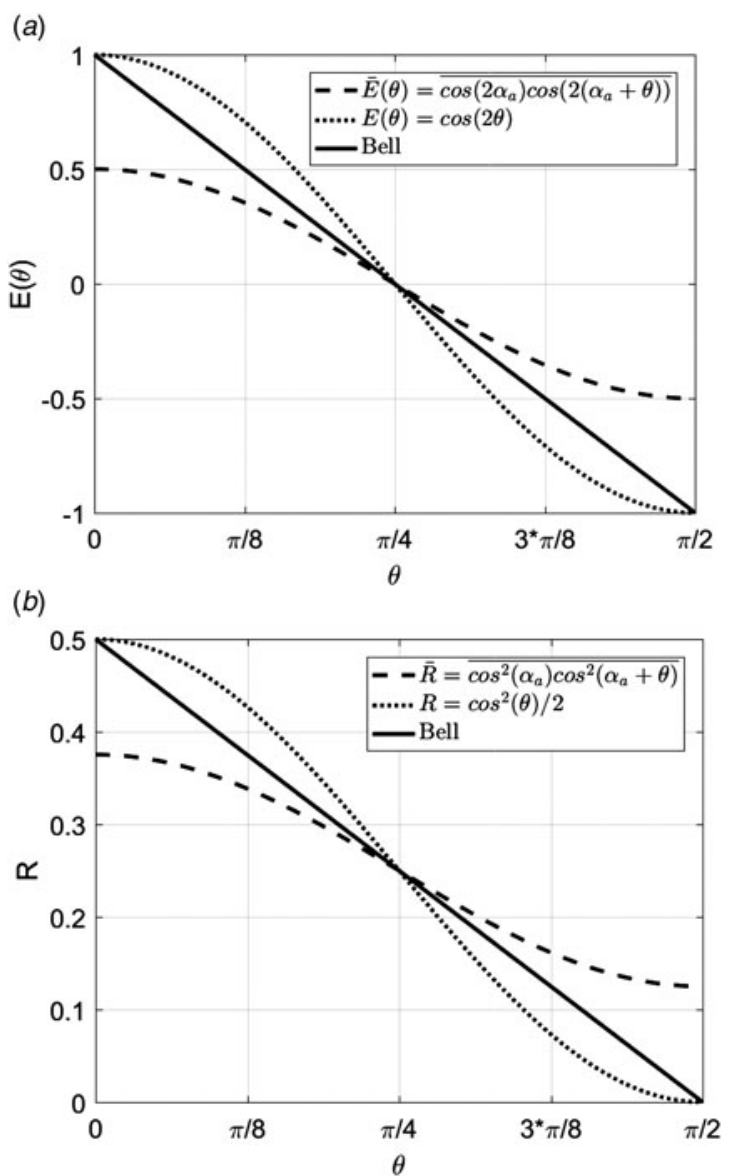

Fig. 1. Photon correlation functions as function of the angle $\theta$ between the polarizer settings, shown together with Bell's inequality (diagonal line). (a) Correlation function $E(\mathbf{a}, \mathbf{b})$ according to the classical and quantum mechanical Eqs. (15) and (16). (b) Correlation function $R(\theta) / R_{0}$ in inequality (17) for classical and quantum mechanical Eqs. (18) and (19).

$\langle E(\mathbf{a}, \mathbf{b})\rangle=\left\langle\cos 2 \alpha_{a} \cos 2 \alpha_{b}\right\rangle$

The biggest difference between the classical and the quantum predictions according to Bell's theorem is noted to be at $\alpha=22.5^{\circ}$ and $\alpha=67.5^{\circ}$ (Clauser et al. 1969). This is so because Bell assumes a linear dependence of the correlation on the angle - see Fig. 1. Setting the a polarizer to $0^{\circ}$ and the b one to $22 \cdot 5^{\circ}$, calculating: $E(\mathbf{a}, \mathbf{b})=2^{-1 / 2}$ and getting $S=E$ $(\mathbf{a}, \mathbf{b})-E\left(\mathbf{a}, \mathbf{b}^{\prime}\right)+E\left(\mathbf{a}^{\prime}, \mathbf{b}\right)+E\left(\mathbf{a}^{\prime}, \mathbf{b}^{\prime}\right)=1.414$ if $\mathbf{a}^{\prime}$ is set at $45^{\circ}$ and $\mathbf{b}^{\prime}$ at $67.5^{\circ}$ as originally suggested, a result that is not in conflict with Bell's inequality. Higher $S$ values can be obtained 'artificially' if other settings of $\mathbf{a}$ and $\mathbf{b}$ are allowed, surmounting to $S=2.828$ for $(\mathbf{a}, \mathbf{b})=\left(\mathbf{a}^{\prime}, \mathbf{b}\right)=\left(\mathbf{a}^{\prime}, \mathbf{b}^{\prime}\right)=$ $\left(0,22 \cdot 5^{\circ}\right)$ in obvious conflict with the Bell inequality, Eq. (9), where $S$ is predicted to be $<+2$ for the classical case. One could question the interpretation of some of these experiments as the delay of the second photon may obstruct entanglement. However, in an experiment by Aspect et al. (1982a) a light switch with $10 \mathrm{~ns}$ period seems to have 
taken care of the latter problem, at least in principle. The corresponding case of orbital angular momentum (circularly polarized photons) may be treated similarly - yielding similar result - using the classical light-polarization vector of Stokes $(1852)$ together with a $(4 \times 4)$ Mueller matrix representing the analyzers (see e.g. Nordén, 1978).

If one assumes that the two photons, or light flashes, are uncorrelated but have the same polarization, which is not unreasonable if they are generated in the same photoselection process by an orthogonal exciting laser beam, Eq. (14) becomes simply:

$\langle E(\mathbf{a}, \mathbf{b})\rangle=(1 / 2) \cos 2 \theta$

(classical)

with $\theta=\left(\alpha_{a}-\alpha_{b}\right)$ the angle between polarizers $\mathbf{a}$ and $\mathbf{b}$. This result is obtained from Eq. (14) by integrating $\langle\cos 2 \alpha \cos 2$ $(\alpha+\theta)\rangle$ over all angles $\alpha$. This classical correlation has the same functional dependence on the angle $\theta$ between the polarizers as the QM correlation:

$\langle E(\mathbf{a}, \mathbf{b})\rangle=\cos 2 \theta$

(QM)

but obviously does not violate the Bell inequality (Fig. 1a). Note, however, that $\eta$ the detection efficiency in Eq. (9) introduces an uncertainty and for less than $\eta=0.83(83 \%$ detection efficiency) $S$ values from Eq. (16) do not any longer violate the Bell inequality.

Historically, one of the first Bell tests, using single channel polarizers (Freedman \& Clauser, 1972), was applying the inequality:

$\Delta=\left|R\left(22.5^{\circ}\right) / R_{0}-R\left(67.5^{\circ}\right) / R_{0}\right| \leqslant 1 / 4$

with $R(\theta)$ the coincidence rate with angle $\theta$ between the polarizers and $\mathrm{R}_{0}$ the rate with polarizers removed. $\Delta=0.35$ was obtained experimentally (Freedman \& Clauser, 1972; Shih \& Alley, 1988), thus clearly violating the inequality. Integrating $\left\langle\cos ^{2} \alpha \cos ^{2}(\alpha+\theta)\right\rangle$ over all angles $\alpha$ gives us the corresponding classically expected correlation function:

$R(\theta) / R_{0}=(1 / 8)(\cos 2 \theta+2) \quad$ (classical)

to be compared with the QM and experimentally observed one (Fig. 1b):

$R(\theta) / R_{0}=(1 / 4)(\cos 2 \theta+1)$

(QM)

Equation (19) reproduces the experimental result $\Delta=0.35$ obtained by Freedman \& Clauser (1972) of Eq. (17). The difference between Eqs. (18) and (19) is $(1 / 8) \cos 2 \theta$, the Bell limit given by $R(\theta)=1 / 2-\theta / \pi$. The similarities between Eqs. (15) and (16) and their relation to the Bell line in Fig. $1 a$, as well as the similarity between Eqs. (18) and (19) are remarkable algebraic features whose interpretation in the respective experimental contexts seems to have been rarely addressed. Ou \& Mandel (1988a) depicted classical and quantum correlation curves like those in Fig. $1 b$.

\section{The 'Interaction Hypothesis'}

The EPR paradox inspired two different strands of experiments, neither of which appears to have given yet an unambiguous answer to the question whether two quantum-entangled particles (or photons) may remain in contact at very large distance from each other so that a collapse of entanglement, induced by interaction with one of them, is immediately propagated to the other one. The first group of experiments were those just mentioned based on the work of Bell (1966); Bohm (1952), Freedman \& Clauser (1972) and others (Aspect et al. 1982a, b; Weihs et al. 1998), focusing on photon statistics and Bell inequality. Fundamental experiments proving the non-locality of our description of the physical world were due to $\mathrm{Wu} \&$ Shaknov (1950) and Wineland (Turchette et al. 1998).

A second line of important experiments were conducted in the interference regime where Mandel and coworkers were pioneers both in method development and to observe nonlocal effects (Gosh \& Mandel, 1987; Hong et al. 1987; Ou \& Mandel, 1988a, b). The discovery by Kimble et al. (1977) of photon anti-bunching in atomic fluorescence was the first example of a new type of phenomena that could not be described without a complete quantum approach. It gave rise to new technologies such as squeezed and entangled light. The work culminated with the first observation of the vacuum Rabi splitting of an atom in the optical domain - basis for a number of applications of quantum logic of single atoms or photons. Here also the demonstrations by Moerner and Orrit of absorption and fluorescence of single molecules trapped in a solid matrix should be mentioned as they led to the also important discovery of antibunching of emitted photons (Basché et al. 1992).

Franson (1989) and Kwiat et al. (1993) have in two-photon interference experiments shown sinusoidal fringes with $80 \%$ visibility, such as predicted by QM, violating Bell inequality by 16 standard deviations. More recently Salart et al. (2008) and Yin et al. (2013), following a proposal by Eberhart (1989) for a 12-h continuous space-like Bell inequality exploiting the Earth's self-rotation, discuss the possibility of superluminal interference at ca $20 \mathrm{~km}$ distance. Already Grangier et al. (1986), using a Mach-Zehnder interferometer and the atom cascade source of Clauser, showed photon anticorrelation effects at a beamsplitter in agreement with QM-described single-photon states. Also using spontaneous parametric downconversion at two wavelengths, a 'heralded' single photon has been used to clock the event when the other was a photon thrown at a beamsplitter after which it is detected in either of two arms of the optics: it is shown that the probability of coincidence in the two detectors $\mathrm{A}$ and $\mathrm{B}, P_{\mathrm{AB}}$ is much less than the probabilities of detection on each side $P_{\mathrm{A}} \times \mathrm{P}_{\mathrm{B}}$, as would be expected in the case of uncorrelated events (Guerreiro et al. 2012). This space-like antibunching experiment beautifully 
corresponds to Albert Einstein's Gedankenexperiment of the 5th Solvay Conference in 1927 and provides strong evidence for the non-locality of a single photon.

For atoms and molecules, quantum entanglement may be viewed as basis of both chemical bonding as well as excitonic stationary quantum states (Kasha 1963; Bayer et al. 2000; Nordén et al. 2010; Halpin et al. 2014). The latter are important in many photophysical and biophysical contexts and can be regarded the result of coupling between molecular subsystems in which virtual excitations via attractive or repulsive interactions lead to eigenstates of the total Hamiltonian, without eigenstates for the separate subsystems. Typically, an excitonic state arising from interactions between degenerate excited states in a dimeric molecular structure may be described by the wave function

$\Psi=2^{-1 / 2}\left[\phi_{\mathrm{A}}^{\circ} \phi_{\mathrm{B}}^{\prime} \pm \phi_{\mathrm{B}}^{\circ} \phi_{\mathrm{A}}^{\prime}\right]$

where $\left|\phi_{\mathrm{A}}{ }^{\circ}\right\rangle$ and $\left|\phi_{\mathrm{A}}{ }^{\prime}\right\rangle$ are, respectively, ground and excited states in fragment $\mathrm{A}$, etc., and eigenstates to the Hamiltonian $H_{\mathrm{A}}$ of that separate fragment. The total excitonic wave function (20) corresponds to entanglement between fragments $\mathrm{A}$ and $\mathrm{B}$ and is an eigenstate of a total Hamiltonian:

$H=H_{\mathrm{A}}+H_{\mathrm{B}}+V_{\mathrm{AB}}$

with $V_{\mathrm{AB}}$ the interaction energy between $\mathrm{A}$ and $\mathrm{B}$. Without entanglement, $V_{\mathrm{AB}}=0$, the exciton $\Psi$ collapses into the product states of the free subsystems, $\left|\phi_{\mathrm{A}}{ }^{\circ}\right\rangle\left|\phi_{\mathrm{B}}{ }^{\prime}\right\rangle$ etc. The impact of the intermolecular interaction $V_{\mathrm{AB}}$ is to ensure indistinguishability of $\mathrm{A}$ and $\mathrm{B}$ as to what molecule actually carries the exciton. In the absence of $V_{\mathrm{AB}}$, symmetry breaking analogous to Jahn-Teller distortion commonly takes place, manifestly localizing the exciton to one of the two molecules. Upon increasing the intermolecular coupling strength though, the relaxation is eventually avoided and the entangled state becomes preferred. If we adopt the same formalism for the entanglement of two photons A and $\mathrm{B}$, the question arises what significance has the interaction operator $V_{\mathrm{AB}}$ ? By analogy with the entangled atom pair, we end up with the following options: either applying the conservation of momentum law for the separate particles or applying the wave function (20) and assigning the interaction $V_{\mathrm{AB}}$ to the entanglement. While the former case is just the rather uninteresting memory effect that we have already discussed for split hydrogen, the latter corresponds to the entanglement that we want to address. For the entanglement between a pair of photons $V_{\mathrm{AB}}$ will be probably the field interaction operator described by quantum electrodynamics and Maxwell's equations. It should satisfactorily explain any experiment performed in laser cavities or other confined optical systems where the photons are in sight' of each other, so we predict, when $V_{\mathrm{AB}}=0$ (or in level with thermal noise), there will be no entanglement. In one stroke this Interaction Hypothesis will dissolve the

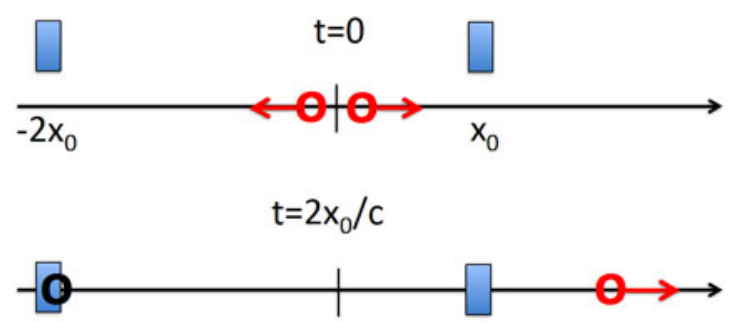

Fig. 2. Proposed experiment to determine whether two photons can be entangled if not 'in sight' of each other (see text).

'spooky-action-at-a-distance' problem as it clearly defines when entanglement can occur but also how fast it may collapse - a change of the fields that connect the photons cannot generally propagate faster than speed of light. ${ }^{2}$

\section{Future experiments}

The author suggests an experiment to decide whether or not photons may be entangled when no longer in field contact with each other (see Fig. 2). Consider a pair of (presumed entangled) photons, one propagating along the positive (right) $x$-axis and one along the negative (left) $x$-axis. They can be produced at a beam-splitter from photon beams of an OPA crystal (Friberg et al. 1985; Kim, 2003; 'heralding' photons may be used to keep track of the photon pair). Introduce then a (black absorbing) beamstop at $x_{0}$, just after that the right-hand photon has passed, and another beamstop at $-2 x_{0}$ just before the left-hand photon is to pass. Will we be able to tell a difference, by probing the right-hand photon, when no longer able of communicating with its left partner, whether the latter is still entangled or has been absorbed? Based on absence of interaction due to beamstop $x_{0}$, one is inclined to say: no!

As far as I know no experiment has yet been performed along this line on single photons, but those experimentalists who pioneered the field of quantum optics could have made it in passing. Kimble has been prolific in squeezed and other quantum states in cavity using the technique of parametric down-conversion and the demonstration by his group (Ou et al. 1992a, b) realizing the EPR paradox for continuous variables using these techniques inspired and paved way for the later establishment of a theory for model-independent continuous-variable entanglements (Duan et al. 2000; Simon, 2000). The group has demonstrated how entangled photons may be created and are not separable. Experiments demonstrating that entangled photon beams may be 'exchanged' into atomic quantum memory, and then mapped back to photonic state again (Choi et al. 2008), will put some special demands on the interaction field hypothesis to be able to adopt to new 'currency' whether photons or

2 A referee argued that an auxiliary field connecting the two photons would have to be in a different Hilbert space in order not to violate the 'monogamy' principle of entangled photon pairs. 
atoms. Besides asking whether our 'interaction postulate' holds, we may also ask: is 'instantaneous' collapse of entanglement in conflict with special relativity? While many theorists have dealt with the tension between QM and relativity, only few experiments have been done. Zbinden et al. (2001) have studied two-photon interference fringes with detectors at $10 \mathrm{~km}$ separation and conclude that introduction of 'passive' detectors (absorbers) does not affect the QM-expected result. Salart et al. (2008) and Yin et al. (2013) as mentioned suggest potential superluminal interference over ca $20 \mathrm{~km}$ distance. Gosh \& Mandel (1987) and Ou \& Mandel (1988a, b) early demonstrated the superiority of interference-beating as a technique for joint two-photon detection. An experiment, representing entanglement of orbital momentum states, should have instead of linearly polarized photons a pair of circularly polarized photons of opposite circularity (Leggett, 2003; Mair et al. 2001). In order to get deeper understanding of the nature of entanglement, systematic studies of dependence on distance and surrounding media are needed - it is also important to quantify the degree of entanglement as has been critically prescribed by Kimble (van Enk et al. 2007).

\section{Conclusions}

The question whether or not single, isolated particles or photons may entangle over large distances remains elusive. The evidence in support of the long-range entanglement hypothesis involves indirect arguments based on photon statistics and conflict with Bell's theorem for classical, compared with quantum logic. A crucial question is how classical properties of a particle system should be defined? It is not quite clear from the assumptions by Bell how 'classical logic' is defined, and the rather unphysical functional appearance in Fig. 1 for the Bell limit could be regarded a source of concern. In my opinion, therefore, some elusiveness remains regarding how the experiments and Bell tests should be performed and interpreted. In addition there could be technical issues: one regarding the true coherence of the two photons created as an entangled pair, another related to detection efficacy.

In order to probe directly the existence of long-range entanglement between two photons, one should first need to create them at the same time (as I suggest, if possible within $10^{-16} \mathrm{~s}$, in contrast to most experiments) and secondly, preferably with similar temporal resolution, determine whether they may dynamically influence each other. One way would be to use interferometry, and here the technique of quantum beating as introduced by Mandel and others appears to be the most promising option. This sets the velocity of light as an upper limit for communication and, trivially, if one photon is affected, the interference will be affected too. It thus remains to prove that entanglement exists in cases where the photons are sufficiently far from each other, and that a perturbation acting on one photon affects the other photon of a pair too, simultaneously or near simultaneously - not only their interference after recombination. To my best knowledge, no experiment has thus far convincingly proven that entanglement can exist between single particles or between single photons when at large distance and completely isolated from each other. Nor has any entanglement experiment provided evidence for the existence of superluminal influence. In other words, except for with single-particle diffraction, there is as yet no direct evidence for the existence of the 'spooky action at a distance' that so concerned Albert Einstein. By way of contrast, manyparticle solutions exist and their entangled states may be prepared along the lines that have been elaborated on by many experimentalists today.

I have discussed the case of entanglement of pairs of atoms, where an ideal model is a diatomic zero-spin molecule that splits in two spin $1 / 2$ atoms. This would be still a testable case in vacuum and absence of fields, whereas spin randomization is anticipated due to interactions with environment or magnetic fields. We also draw attention to the possible importance of temperature. While the long-range entanglement still remains to be proven for material particles, there are many successful experiments evidencing their quantum nature (Rowe et al. 2001; Turchette et al. 1998).

The discussion in this paper should not be confused with applications of quantum theory to microscopic systems, where it is well established that, e.g. spins of single electrons may be probed and exploited in solid-state devices. Entanglement is, thus, not an issue in microscopic systems, such as the intact $\mathrm{H}_{2}$ molecule. Stationary entanglement is also an important concept in interacting aggregates, excitonic states in molecules being an example. This entanglement can be regarded as the coupling between subsystems in which virtual excitations via attractive or repulsive interactions lead to eigenstates of the total Hamiltonian, but without eigenstates for the separate subsystems. Superconductivity can be treated also along these lines as shown by Panas (2015). For all these cases, though, in absence of any interaction between the components, entanglement does not exist.

In contrast to the two-photon and two-particle entanglements that this paper focuses on, and where elusiveness seems to remain, recent experiments showing space-like antibunching of single photons have been reported related to Einstein's 1927 original Solvay Gedanken diffraction experiment (Guerreiro et al. 2012; Takeda et al. 2013). Indeed they confirm non-locality, i.e. how a single photon may deposit itself in either of several different locations. It has also been demonstrated that affecting the outcome in one location will influence that of another according to the energy conservation principle (Fuwa et al. 2015). However, it should be 
noted that the photon is confined inside an optical cavity or waveguide so the fields can communicate back and forth (by velocity of light). The 'interaction hypothesis' is thus not violated. Along the same line we have suggested an experiment that might judge whether or not photons may be entangled when not being in field contact with each other: introducing a beam stop after that one of the photons of an entangled pair has passed should, we propose, inhibit entanglement, and thus confirm the interaction postulate.

The EPR entanglement concept connects to another Einstein land-mark paper of 1935 (Einstein \& Rosen, 1935) involving general relativity. An amazing new direction of research, inspired by those papers, has evolved suggesting that entanglement of quantum fields at the boundary of a given region are responsible for the very emergence of space and time in that region (Cowen, 2015). Entanglement is proposed to be a 'geometric glue' of spacetime and between QM and gravity, with spectacular potential consequences including wormholes between entangled black holes, parallel universa etc. (Gefter, 2014; Maldacena \& Susskind, 2013; Van Ramsdonk, 2010).

\section{Acknowledgements}

I am grateful to Professors A. D. Buckingham, C. Dreismann, P. Pyykkö, I. Panas and H. Wennerström for inspiring discussions and valuable comments on the manuscript. I am grateful for support from the Swedish Research Council (VR).

\section{References}

Ardavan, A, Rival, O, Morton, J. L., Blundell, S., Tyryshikin, Timco, G. A. \& WinpenNY, E. P. (2007). Will spin relaxation in molecular magnets permit quantum information processing? Physical Review Letters 98, 57201-57204.

Aspect, A., Dalibard, J. \& Roger, G. (1982a). Experimental test of Bell's inequalities using time-varying analyzers. Physical Review Letters 49, 1804-1807.

Aspect, A., Grangier, P. \& Roger, G. (1982b). Experimental realization of Einstein-Podolsky-Rosen-Bohm Gedankenexperiment: a new violation of Bell's inequlities. Physical Review Letters 49, 91-94.

Atkins, P. W., Mclauchlan, J. \& Percival, P. W. (1973). Electron spin-lattice relaxation times from the decay of e.s.r. emission spectra. Molecular Physics 25, 281-296.

Ballentine, L.E. (1970). The statistical interpretation of quantum mechanics. Reviews of Modern Physics 42, 358-381.

Basché, T. H., Moerner, W. E., Orrit, M. \& Talon, H. (1992). Photon antibunching in the fluorescence of a single dye molecule trapped in a solid. Physical Review Letters 69, 1516-1519.

Bayer, M., Hawrylak, P., Hinzer, K., Fafard, S., Korkusinsky, M., Wasilewki, Z. R., Stern, O. \& Forchel, A. (2000). Coupling and entangling of quantum states in quantum dot molecules. Science 291, 451-453.
BELL, J.S. (1966). On the problem of hidden variables in quantum mechanics. Reviews of Modern Physics 38, 447-452.

Bennett, C. H., Brassard, G., Crepenau, C., Jozsa, R., Peres, A. \& Wootters, W. K. (1993). Teleporting an unknown quantum state via dual classical and Einstein-Podolsky-Rosen channels. Physical Review Letters 70, 1895-2001.

Blatt, R. \& Wineland, D. (2008). Entangled states of trapped atomic ions. Nature 453, 1008-1015.

Bogani, L. \& WeRNSDORFER, W. (2008) Molecular spintronics using single-molecule magnets. Nature Materials 7, 179-186.

Вонм, D. (1952). A suggested interpretation of the quantum theory in terms of hidden variables. Physical Review 85, 166-179.

Bohm, D. \& Aharonov, Y. (1957) Discussion of experimental proof for the paradox of Einstein, Podolsky and Rosen. Physical Review 108, 1070-1076.

BoHR, N. (1935a). Can quantum-mechanical description of physical reality be considered complete? Physical Review 48, 696-702.

BoHR, N. (1935b). Quantum mechanics and physical reality. Nature 136, 65-65.

Bransden, B. H. \& Joachain, C. (2000). Quantum Mechanics, 2nd edn, pp. 769-770. New York: Prentice Hall.

Carg, A. \& Mermin, N.D. (1987). Detector inefficiencies in the Einstein-Podolsky-Rosen experiment. Physical Review D 35, 3831-3835.

Choi, K. S., Deng, H., Laurat, J. \& Kimble, H. J. (2008) Mapping photonic entanglement into and out of quantum memory. Nature 452, 67-71.

Clauser, J. F. \& Horne, M. A. (1974). Experimental consequences of objective local theories. Physical Review D 10, 526-535.

Clauser, J. F. \& Shimony, A. (1978). Bell's theorem: experimental tests and implications. Reports on Progress in Physics 41, 1881-1927.

Clauser, J. F., Horne, M. A., Shimony, A. \& Holt, R. A. (1969). Proposed experiment to test local hidden-variable theories. Physical Review Letters 23, 880-884.

Cowen, R. (2015). Space. Time. Entanglement. Nature 527, 290-293.

Dirac, P.A.M. (1926). On the theory of quantum mechanics. Proceedings of the Royal Society of London, Series A 112, 661-677.

Duan, L-M., Giedke, G., Cirac, J. I. \& Zoller, P. (2000). Entanglement purification of Gaussian continuous variable quantum states. Physical Review Letters 84, 4002-4005.

Eberhart, P. H. (1989) Quantum Theory and Pictures of Reality, pp. 169-216. Berlin: Springer.

Einstein, A. (1936). Physik und Realität. Journal of Franklin Institute 221, 313-347.

Einstein, A. \& Rosen, N. (1935). The particle problem in the general theory of relativity. Physical Review 48, 73-77.

Einstein, A., Podolsky, B. \& Rosen, N. (1935). Can quantummechanical description of physical reality be considered complete? Physical Review 47, 770-780.

EKERT, A. (1991). Quantum cryptography based on Bell's theorem. Physical Review Letters 67, 661-663.

Engel, V., Metiu, H., Almeida, R., Marcus, R. A. \& Zewail, A. H. (1988). Molecular state evolution after excitation with an ultrashort laser pulse. A quantum analysis of $\mathrm{NaI}$ and $\mathrm{NaBr}$ dissociation. Journal of Chemical Physics 90, 6116-6128.

Epstein, R. J., Mendoza, F. M., Kato, Y.K. \& Awschalom, D. D. (2005). Anisotropic interactions of single spin and dark-spin spectroscopy in diamond. Nature Physics 1, 94-98. 
Franson, J. D. (1989) Bell inequality for position and time. Physical Review Letters 62, 2205-2208.

Freedman, S. J. \& Clauser, J. F. (1972). Experimental test of local hidden variable theories. Physical Review Letters 28, 938-941.

Friberg, S. R., Hong, C. K. \& Mandel, L. (1985) Measurement of time delays in the parametric production of photon pairs. Physical Review Letters 54, 2011-2013.

Fuwa, M., Takeda, S., Zwierz, M., Wiseman, H. \& Furusawa, A. (2015). Experimental proof of nonlocal wavefunction collapse for a single particle using homodyne measurements. Nature Communication 6, 1-6. doi: 10.1038/ncomms 7665.

Gefter, A. (2014). Theoretical physics: complexity on the horizon. Nature 509, 552-553.

Gisin, N. \& Thew, R. (2007). Quantum communication. Nature Photonics 1, 165-171.

Giustina, M., Versteegh, M. A., Wengerowsky, S., Handsteiner, J., Hochrainer, A., Phelan, K., Steinlechner, F., Kofler, J., Larsson, J.-Å., Abellan, C., Amaya, W., Pruneri, V., Mitchell, M. W., BeVer, J., Gerrits, T., Lita, A. E., Shalm, L. K., Nam, S. W., Scheidl, T., Ursin, R., Wittman, B. \& Zeilinger, A. (2015). Significant loophole-free test of Bell's theorem with entangled photons. Physical Review Letters 115, 250401-250406.

Gosh, R. \& MANDEL, L. (1987). Observation of nonclassical effects in the interference of two photons. Physical Review Letters 59, 19031905.

Grangier, P., Roger, G. \& Aspect, A. (1986). Experimental evidence for a photon anticorrelation effect on a beamsplitter: a new light on single-photon interferences. Europhysics Letters 1, 173-179.

GRIFFITHS, D. J. (2008). Introduction to Quantum Mechanics, 2nd edn, pp. 207-210. New Jersey: Wiley-VCD.

Guerreiro, T., Sanguinetti, B., Zbinden, H., Gisin, N. \& Suarez, A. (2012). Single-photon space-like antibunching. Physics Letters A 376, 2174-2177.

Halpin, A., Johnson, P. J. M., TempelaAr, R., Murphy, R. S., Knoester, J., Jansen, T. L.C. \& Miller, R. J.D. (2014). Two-dimensional spectroscopy of a molecular dimer unveils the effects of vibronic coupling on exciton coherences. Nature Chemistry 6, 196-201.

Hensen, B., Bernien, H., Dreau, A. E., Reiserer, A., Kalb, N., Blok, M. S., Ruttenberg, J., Vermeulen, R. F. L., Shouten, R. N., Abellán, C., Maya, M., Pruneri, V., Mitchell, M. W., Markham, M., Twitchen, D. J., Elkouss, D., Wehner, S., TAminiaU, T. H. \& Hanson, R. (2015). Loophole-free Bell inequality violation using electron spins separated by 1.3 kilometres. Nature 526, 682-686.

Hong, C. K., Ou, Z. Y. \& MANDeL, L. (1987). Measurement of subpicosecond time intervals between two photons by interference. Physical Review Letters 59, 2044-2046.

Horne, M. A. \& Zeilinger, A. (1985). A Bell type EPR experiment using linear momenta. In Proc. Symp. on Found. of Mod. Physics (eds. P. Lahiti \& P. Mittelstaedt), p. 435. World Scientific, Singapore.

Jammer, M. (1974). The Philosophy of Quantum Mechanics, p. 115. Singapore: John Wiley.

Johnson, A. C., Petta, J. R., Taylor, J. M., Yacoby, A., Lukin, M. D., Marcus, C. M., Hanson, M.P. \& Gossard, A.C. (2005) Triplet-singlet spin relaxation via nuclei in a double quantum dot. Nature 435, 925-928.

KASHA, M. (1963). Energy transfer mechanisms and the molecular exciton model for molecular aggregates. Radiation Research 20, $55-71$.
Kemble, E. C. (1935). The correlation of wavefunctions with the state of physical systems. Physical Review 47, 973-974.

KIM, Y.-H. (2003). Two-photon interference without bunching two photons. Physics Letters A 315, 352-357.

Kimble, H. J., Dagenais, M. \& Mandel, L. (1977). Photon Antibunching in Resonance Fluorescence. Physical Review Letters 39, 691-695.

Kocher, C. A. \& Commins, E. D. (1967). Polarization correlation of photons emitted in an atomic cascade. Physical Review Letters 18, 575-579.

Kwiat, P. G., Steinberg, A. M. \& Chiao, R. Y. (1993). High visibility interference in a Bell-inequality experiment for energy and time. Physical Reviews A 47, 2472-247.

LARSSON, J.-Å. (1998). Detector efficiency in the Greenberger-Horne-Zeilinger paradox: independent errors Physical Reviews A 57, 3303.

LegGetT, A. J. (2002). Testing the limits of quantum mechanics: motivation, state of play, prospects. Journal of Physics: Condensed Matter 14, R415-R451.

LEgGetT, A. J. (2003). Nonlocal hidden-variable theories and quantum mechanics: an incompatibility theorem. Foundations of Physics 33, 1469-1493.

Mair, A., Vaziri, A., Weins, G. \& Zeilinger, A. (2001). Entanglement of the orbital angular momentum states of photons. Nature $\mathbf{4 1 2}$ 313-316.

Maldacena, J. \& Susskind, L. (2013). Cool horizons for entangled black holes. Progress of Physics 61, 781-811.

NinHAM, B. W. \& DaICIC, J. (1998). Lifshitz theory of Casimir forces at finite temperature. Physical Reviews A 57, 1870-1877.

NORDÉN, B (1978). Applications of Linear Dichroism spectroscopy. Applied Spectroscopy Reviews 14, 157-248; Appendix C.

Nordén, B., Elvingson, C., Jonsson, M. \& Åkerman, B. (1991). Microscopic behaviour of DNA during electrophoresis: electrophoretic orientation. Quarterly Reviews of Biophysics 24, 103-164.

Nordén, B., Rodger, A. \& Dafforn (2010). Linear Dichroism and Circular Dichroism. A Textbook on Polarized-light Spectroscopy, pp. 240-250. Lomdon: The Royal Society of Chemistry.

Ou, Z. Y. \& MANDEL, L. (1988a). Violation of Bell's inequality and classical probability in a two-photon correlation experiment. Physical Review Letters 61, 50-53.

Ou, Z. Y. \& MANDEL, L. (1988b). Observation of spatial quantum beating with separated photodetectors. Physical Review Letters 61, 54-57.

Ou, Z. Y., Pereira, S. F., Kimble, H. J. \& Peng, K. C. (1992a). Realization of the Einstein-Podolsky-Rosen paradox for continuous variables. Physical Review Letters 68, 3663-3666.

Ou, Z. Y., Pereira, S. F. \& Kimble, H. J. (1992b). Realization of the Einstein-Podolsky-Rosen paradox for continuous variables in non-degenerate parametric amplification. Applied Physics B 55, 265-278.

PANAS, I. (2015). A tale of two entangled instabilities - dual role of delta oxygen in $\mathrm{HgBa}_{2} \mathrm{Can}-1 \mathrm{CunO}_{2}(\mathrm{n}+1)+\delta$. Entropy 17, 67656782.

Rose, T. S., Rosker, M. J. \& ZewaIL, A. H. (1988). Femtosecond real time observation of wave packet oscillations (resonance) in dissociation reactions. Journal of Chemical Physics 88, 6672-6673.

Rowe, M. A., Kielpinski, D., Meyer, V., Sackett, C. A., Itano, W. M., Monroe, C. \& Wineland, D. J. (2001). Experimental violation of a Bell's inequality with efficient detection. Nature 409, 791-794. 
Salart, D., BaAs, A., Branciard, C., Gisin, N. \& Zbinden, H. (2008). Testing the speed of 'spooky action at a distance'. Nature 454, 861-864.

SCHRÖDINGER, E. (1935). Discussion of probability relations between separated systems. Proceedings of the Cambridge Philosophical Society 31, 555-563.

Shit, Y.H. \& Alley, C.O. (1988). New type of Einstein-Podolsky-Rosen-Bohm experiment using pairs of light quanta produced by optical parametric down conversion. Physical Review Letters 61, 2921-2924.

Simon, R. (2000). Peres-horodecki separability criterion for continuous variable systems. Physical Review Letters 84, 2726-2729.

SтокеS, G. G. (1852). Optical parameters for polarized light. Transactions of the Cambridge Philosophical Society 9, 399-415.

Takeda, S., Mizuta, T., Fuwa, M., Yoshikawa, J.-I., Yonesawa, H. \& FurUSAWA (2013). A generation and eight-port homodyne characterization of time-bin qubits for continuous-variable quantum information processing. Physical Reviews A 87, 043803/1-5.

Turchette, Q. A., Wood, C. S., King, B. E., Myatt, C. J., Leibfried, D., Itano, W. M., Monroe \& Wineland, D. J. (1998). Deterministic entanglement of two trapped ions. Physical Review Letters 81, 3631-3634.

VAn EnK, S. J., Luthenhaus, N. \& Kimble, H. J. (2007). Experimental procedures for entanglement verification. Physical Review A 75, $1-15$.

VAN RAmsdonK, M. (2010). Building up spacetime with quantum entanglement. General Relativity and Gravitation 42, 2323-2329.

VAN VleCK, J.H. (1932). Electric and Magnetic Susceptibilities, p. 318. Oxford: Clarendon Press.

Vaterlaus, A., Beutler, T. \& Mair, F. (1991). Spin-lattice relaxation time of ferromagnetic gadolinium determined with time-resolved spin-polarization photo-emission. Physical Review Letters 67, 3314-3317.

Weins, G., Jennewein, T., Simon, C., Weinfurter, H. \& Zeilinger, A. (1998). Violation of Bell's inequality under strict Einstein locality conditions. Physical Review Letters 81, 5039-5046.

Wennerstrom, H., Daicic, J. \& Ninham, B. W. (1999). Temperature dependence of atom-atom interactions. Physical Review A 60, 2581-2584.

Wennerström, H. \& Westlund, P.-O. (2013). On Stern-Gerlach coincidence measurements and their application to Bell's theorem. Physics Essays 26, 174-180.

White, R. M. (2007). Quantum Theory of Magnetisms: Magnetic Properties of Materials. Berlin: Springer-Verlag, Section 2.2.7.

Wratchtrup, J., von Borczyskowski, C., Bernard, J., Orrit, M. \& Brown, R. (1993). Optically detected spin coherence of single molecules. Physical Review 71, 3565-3568.

Wu, C. S. \& SHAKNov, I. (1950). The angular correlation of scattered annihilation radiation. Physical Review 77, 136.

Yin, J., CaO, Y., Yong, H.-L., Ren, J.-G., Linag, H., LiaO, S.-K., Zhou, F., LiU, C., Wu, Y.-P., Pan, G.-S., Zhang, Q., Peng, C.-Z. \& Pan, J.-W. (2013). Bounding the speed of 'spooky action at a distance'. Physical Review Letters 110, 260407-260418.

ZAGer, S. A. \& FreED, J. H. (1982). Electron spin relaxation and molecular dynamics in liquids: density dependence. Journal of Chemical Physics 77, 3360-3375.

Zinden, H., Brendel, J., Gisin, N. \& Tittel, W. (2001) Experimental test of nonlocal quantum correlation in rekativistic configurations. Physical Review A 63, 022111-1-10.

ZeWAIL, A. H. (1988). Laser femtochemistry. Science 242, 1645-1653.

Zewall, A. H. (1990). The birth of molecules. Scientific American 263, 76-86. 